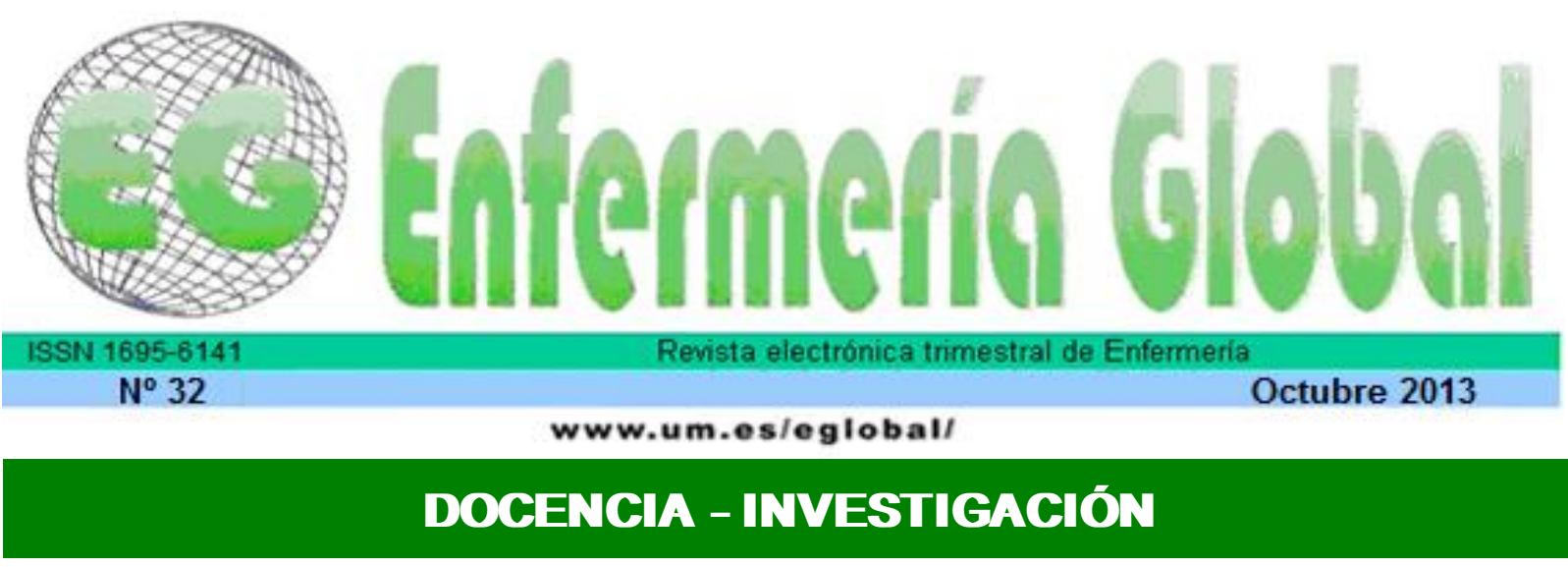

\title{
El Conjunto Mínimo Básico de Datos como Herramienta de Vigilancia Epidemiológica de la Incidencia Diabetes Mellitus 1: Experiencia en Extremadura
}

The Minimum Basic Data Set as an Epidemiological Vigilance tool for the Incidence of Diabetes Mellitus 1 (DM 1): Experience in Extremadura

\section{*Gimeno Benítez, Alfredo *Suero Villa, Pedro ${ }^{* *}$ Suero Villa, Severo ${ }^{* *}$ Guerrero Porras, Maruja ${ }^{* *}$ Sánchez Veja, Javier *Luengo Pérez, Luis Miguel}

*SES (Servicio Extremeño de Salud). UEX (Universidad de Extremadura). E-mail: agimben@unex.es **SES (Servicio Extremeño de Salud). Badajoz.

Palabras clave: CMBD; Calidad asistencial; Epidemiología

Keywords: CMBD; care quality; epidemiology

\section{RESUMEN}

El 'gold standard' del conocimiento de la evolución epidemiológica de las patologías que para su diagnóstico necesitan hospitalización, son las historias clínicas, aunque la recogida de datos de ella es lenta, dificultosa y costosa en tiempo, por lo que si validáramos otra fuente de datos que fuese más rápida, fácil y barata sería de gran utilidad para los estudios epidemiológicos.

Objetivo. Verificar la validez del el Conjunto Mínimo Básico de Datos (CMBD) como fuente de vigilancia epidemiológica, comparando los datos del CMBD y los recogidos en la historia clínica.

Metodología. Comparamos los datos del CMBD con los registros de las historias clínicas de los casos de incidencias de la Diabetes Mellitus 1 (DM 1) en la población menor de 15 años de Extremadura durante 2003-2007 en un estudio observacional retrospectivo y descriptivo, con método de captura-recaptura.

Resultados. Los casos seleccionados fueron 208. En el 2,40 \% de los casos no coincide con la codificación de diabetes. En un 6,73\% de los casos en el CMBD están codificados con otros tipos de diabetes u otra patología. De los codificados como DM 1 con cetoacidosis, el 20,75\% no consta cetoacidosis en la historia, y en el 69,71\% coinciden historia y el CMBD.

Discusión. A pesar de sus limitaciones, el CMBD ha demostrado ser una fuente de información valiosa.

Conclusión. EI CMBD sirve como fuente de información, pero no como vigilancia de la epidemiológica de la DM 1 


\section{ABSTRACT}

The 'gold standard' of knowledge of the epidemiological evolution of the diseases that require hospitalization for diagnosis are the medical records, although the data collection is slow, difficult and time consuming, so if another faster, easier and cheaper source of data was validated, it would be very useful for epidemiological studies.

Objectives. To verify The Minimum Basic Data (CMBD) validity as a source of epidemiological vigilance, comparing the $\mathrm{CMBD}$ data to the collected information in the medical record.

Methodology. The CMBD data compared with the records of the medical records of incident cases of type 1 diabetes mellitus (DM 1) in the population under 15 years of Extremadura during 2003-2007 in a retrospective observational study and descriptive with capture-recapture method.

Results. The cases were selected from 208. In $2.40 \%$ of cases, the results do not coincide with diabetes coding. $6.73 \%$ of cases, the results in the CMBD are coded with other types of diabetes or other pathology. $20.75 \%$ of the coded cases as DM 1 in with ketoacidosis doesn't have ketoacidosis in its history, and $69.71 \%$ the medical history matches with the CMBD.

Discussion. Despite of its limitations, the CMBD has proved to be a valuable source of information.

Conclusion. The CMBD serves as a source of information, but not as DM 1 in epidemiological surveillance

\section{INTRODUCCIÓN}

El 'gold standard' del conocimiento de la evolución epidemiológica de las patologías que para su diagnóstico necesitan hospitalización, son las historias clínicas, aunque la recogida de datos de ella es lenta, dificultosa y costosa en tiempo, por lo que si validáramos otra fuente de datos que fuese más rápida, fácil y barata sería de gran utilidad para los estudios epidemiológicos.

El Conjunto Mínimo Básico de Datos (CMBD) fue la respuesta a la necesidad de recoger de manera sistemática unos datos básicos del proceso asistencial del paciente, con el objetivo de disponer de un sistema de información homogénea, exhaustiva y de uniformidad en la calidad y que fuese un instrumento útil tanto para los profesionales sanitarios como a los gestores.

Representa en definitiva, aquella información básica cuya necesidad es común a diferentes usuarios (clínicos, epidemiológicos, etc.) aunque, evidentemente, resulta insuficiente para cada uno de ellos $y$, como puede observarse, las variables de carácter sanitario (diagnóstico principal, otros diagnósticos, procedimientos), son las que tienen mayor interés para ser utilizadas como instrumento en la evaluación de la actividad sanitaria ${ }^{1}$.

La base de datos administrativa que recoge el CMBD incorpora la codificación de la novena revisión de la Clasificación Internacional de las Enfermedades (CIE-9-MC) para diagnósticos y procedimientos terapéuticos, quirúrgicos y obstétricos que figuran en el informe médico al alta. Es de uso obligado y común en todos los hospitales públicos de España y fue creada y promocionada desde el Ministerio de Sanidad y Consumo ${ }^{2}$. 
Su uso sigue siendo fundamentalmente administrativo con fines de gestión, control y planificación hospitalaria, pero cada vez más empieza a usarse con fines de investigación epidemiológica al recoger datos de persona, lugar y tiempo de cualquier proceso asistencial ${ }^{3}$.

Se cumplimenta, habitualmente, por personal no sanitario, lo que puede ser su principal limitación, al generar dificultades de interpretación, aumentando el riesgo de error en la codificación.

En una publicación 1998 se demostró que había una alta calidad en la cumplimentación de los datos administrativos, pero existían problemas de calidad en la codificación de la información clínica, afectando a la validez de los estudios epidemiológicos ${ }^{4}$.

\section{OBJETIVO}

Verificar la validez del CMBD como fuente de vigilancia epidemiológica para la Diabetes Mellitus 1 (DM 1) en la Comunidad Autónoma de Extremadura.

\section{METODOLOGÍA}

Comparamos los datos del CMBD con los registros de las historias clínicas de los casos de incidencias de la Diabetes Mellitus 1 (DM 1) en la población menor de 15 años de Extremadura durante 2003-2007 en un estudio observacional retrospectivo y descriptivo, con método de captura-recaptura.

Utilizamos como fuente primaria de datos el CMBD de los hospitales de Extremadura para la DM 1. Para identificar los casos en el CMBD se seleccionaron aquellos pacientes en los que figurase codificado como diagnóstico principal o secundario los relacionados con la diabetes mellitus, código 250 . Los pacientes son identificados a través del Código de Identificación Personal (CIP).

Como fuente secundaria explotamos los datos de facturación de recetas médicas del Servicio Extremeño de Salud correspondiente al subgrupo correspondiente a insulinas, A10A de la ATC (Anatomical, Therapeutic, Chemical Classification System) ${ }^{5}$

Para determinar el grado de exhaustividad utilizaremos el método captura-recaptura modificado por Chapman y Seber ${ }^{6,7}$.

La población de estudio son los menores de 15 años de la Comunidad Autónoma de Extremadura según los datos del Instituto Nacional de Estadística.

Tendrá consideración de caso todo paciente menor de 15 años, diagnosticado de DM 1 entre el 1 de enero 2003 hasta 31 diciembre 2007, residentes en la Comunidad Autónoma de Extremadura al menos en los últimos 6 meses previos, haber ingresado en su debut en un hospital de la Comunidad Autónoma o que durante el periodo de estudio haber recibido prestación farmacéutica de algún fármaco del subgrupo $\mathrm{A} 10 \mathrm{~A}$ de la ATC a través de receta oficial.

Se excluyen otros tipos de diabetes, haber recibido prestación farmacéutica de algún fármaco del subgrupo A10A de la ATC a través de receta oficial con anterioridad al periodo de estudio. 
Verificados los criterios de inclusión, y en situaciones de ingresos repetidos, consideramos para el estudio los datos del primer ingreso.

\section{RESULTADOS}

De los 655 registros identificados del CMBD, tras verificar los reingresos y pacientes con varios números de historia por ingresos en diferentes hospitales de la Comunidad, se obtienen 326 casos. En un 34,6\% no constaba el CIP, por lo que para obtenerlo y no perderlo como caso, se utilizó otra fuente HP-HIS, que es una adaptación de Hewllet-Packard Iberica personalizada del sistema de información para todos los hospitales del Instituto Nacional de la Salud (INSALUD).

Los registros eliminados y sus causas se muestran en la tabla I.

\begin{tabular}{|lc|}
\hline \multicolumn{1}{|c|}{$\begin{array}{lc}\text { Mabla I. Registros Excluidos } \\
\text { Motivo exclusión }\end{array}$} & N \\
No eran incidentes & 74 \\
Otros tipos de diabetes o hiperglucemias ocasionales & 7 \\
Historias no localizadas & 5 \\
No diagnosticados de diabetes & 3 \\
No residencia en CCAA & 7 \\
Por historia clinica en varias Áreas de salud & 22 \\
\hline
\end{tabular}

Se identifican 208 casos incidentes de DM 1. En la fuente secundaria se identificaron 190 y de ellos el $100 \%$ de los casos estaban en la fuente principal (tabla II).

\begin{tabular}{|lccccccc|}
\hline \multicolumn{1}{c}{ Fuente } & \multicolumn{7}{c|}{ Tabla II Casos por año } \\
& 2003 & 2004 & 2005 & 2006 & 2007 & Total casos \\
CMBD & 39 & 42 & 41 & 47 & 39 & 208 \\
Consumo Farmacia & 36 & 39 & 37 & 42 & 46 & 190 \\
\hline
\end{tabular}

El grado de exhaustividad de la fuente principal es el $100 \%$ y de la secundaria el $91,34 \%$, por lo tanto el grado de exhaustividad del estudio es del $100 \%$. Con cualquiera de las dos fuentes por si sola podrían dar validez al trabajo.

En el $2,40 \%$ de los casos el código principal no coincide con la codificación de diabetes (tabla III). 


\begin{tabular}{|llcc|}
\hline \multicolumn{1}{|c}{$\begin{array}{c}\text { Tabla III. Casos según codificación CMBD } \\
\text { CóDIGo CIE 9 MC }\end{array}$} & $\begin{array}{c}\text { Sin } \\
\text { cetoacidosis }\end{array}$ & $\begin{array}{c}\text { Con } \\
\text { cetoacidosis }\end{array}$ \\
250.00 & Diabetes $m$. Sin mención compl. Tipo II o no esp. No establecida como incontrolada & 3 & 3 \\
250.01 & Diabetes m. Sin mencion compl. Tipo I no indicada como incontrolada & 91 & 36 \\
250.03 & Diabetes m. Sin mencion compl. Tipo I incontrolada & 2 & 1 \\
250.10 & Diabetes con cetoacidosis tipo II o no esp. No establecida como incontrolada & 1 & 1 \\
250.11 & Diabetes con cetoacidosis tipo I no indicada como incontrolada & 10 & 40 \\
250.12 & Diabetes con cetoacidosis tipo II o no esp. Incontrolada & 0 & 1 \\
250.13 & Diabetes con cetoacidosis tipo I incontrolada & 1 & 1 \\
250.31 & Diabetes con otro coma, tipo I no indicada como incontrolada & 0 & 1 \\
250.41 & Diabetes con Manis. Renales tipo I no indicada como incontrolada & 1 & 0 \\
250.81 & Diabetes con otras Manis. Tipo I no descompensada & 5 & 3 \\
250.83 & Diabetes con otras Manis. Tipo I descompensada & 1 & 0 \\
250.91 & Diabetes compl. No esp. Tipo I no descompensada & 1 & 0 \\
Otros & Codificados con DM en diagnóstico secundario o terciario & 4 & 1 \\
\hline
\end{tabular}

En un $6,73 \%$ de los casos identificados como DM 1 en la historia clínica, en el CMBD figura como código principal otros tipos de diabetes, o código principal asociado a otra patología y el secundario o terciario como diabetes.

De los casos codificados como DM 1 con cetoacidosis, tras valorar las historias clínicas, el $20,75 \%$ no presentan cetoacidosis.

Atendiendo a la presencia o no de complicaciones, cetoacidosis y control de la enfermedad, en el $69,71 \%$ es coincidente la situación clínica con la codificación principal.

\section{DISCUSIÓN}

A pesar de sus limitaciones el CMBD ha demostrado ser una fuente de información valiosa y que puede usarse con fines descriptivos y evaluadores de la práctica médica $^{8-14}$.

El porcentaje de error detectado entre el código principal y la situación clínica en la DM 1 en Extremadura, 30,29\%, coincide con los primeros trabajos realizados en EE.UU. sobre el Uniforma Hospital Discare Data Sep (UHDSS) que es considerado el origen del CMBD, donde encontraron tasas de error en el diagnóstico principal respecto al que constaba en la historia clínica, superiores al 30\% para todo tipo de patologías ${ }^{15-17}$. Estudios posteriores realizados en ese mismo país muestran proporciones de error en torno al $20 \%{ }^{18,19}$.

En España aportaron resultados muy parecidos (24.9\%), en el estudio donde analizaron calidad de las variables administrativas del CMBD de Osakidetza, Servicio Vasco de Salud en el $2008^{20 .}$

Los intervalos de error son diferentes según los diagnósticos (variaban desde un $2 \%$ para la neumonía hasta un $45 \%$ en el infarto de miocardio) o los hospitales. No podemos establecer comparativa con la DM 1 al no encontrar estudios documentados sobre esta patología ${ }^{21,22}$. 
EI CMBD es un instrumento que contiene un gran volumen de información fácilmente disponible y eficiente para el análisis de resultados, aunque hay que tener en cuenta sus limitaciones al analizar los datos, por lo que es especialmente útil en combinación con otros métodos de investigación para valorar diferentes aspectos de la práctica

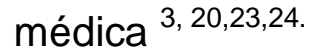

\section{CONCLUSIÓN}

Podemos concluir que el Conjunto Mínimo Básico de Datos es una buena fuente de información en el estudio de la incidencia de la Diabetes Mellitus 1 en Extremadura al obtener, como fuente principal, un grado de exhaustividad del $100 \%$.

Como vigilancia de la evolución epidemiológica de la incidencia de la Diabetes Mellitus 1, al tener un 30,29\%, de errores en la codificación, no es una herramienta útil.

\section{BIBLIOGRAFÍA}

1. Ministerio de Sanidad y Consumo, Explotación de bases del CMBD. Estadísticos de referencia estatal de los sistemas de agrupación de registro de pacientes. Madrid 1995.

2. Edición electrónica de la CIE-9-MC, 8a Edición (Enero 2012), Versión 1.1 .0 15/12/2011 [Consultado 11-02-2012]. Disponible en: http://eciemaps.mspsi.es/ecieMaps/browser/index_9_2012.html.

3. Sendra JM, Sarria-Santamera A, Iñigo J, et al. Factores asociados a la mortalidad intrahospitalaria del infarto de miocardio. Resultados de un estudio observacional. Med Clin (Barc). 2005; 125:641-6.

4. Librero J, Ordinana R, Peiro S. Análisis automatizado de la calidad del conjunto mínimo de datosbásicos. Implicaciones para los sistemas de ajuste de riesgos. Gac Sanit. 1998; 12:9-21.

5. WHO Collaborating Centre for Drug Statistics Methodology. Updates included in the ATC/DDD Index. [consultado el 12 de junio de 2013] Disponible en: http://www.whocc.no/atc_ddd_index/?code=A10.

6. Chapman DG. Some properties of the hypergeometric distribution with applications to zoologicalsimple censuses. Univ Calif Public. Stat, 1951; 1:131-60. Comentario en: Am J Epidemiol. 1992; 135:1060-7.

7. Seber GAF. The effect of trap response on tag recapture estimates. Biometrics. 1970; 26:13-22.

8. Barba R, Losa JE, Velasco M,et al. Mortalidad en los pacientes ingresados durante los fines de semana. Med Clin (Barc). 2005; 125:784-9.

9. Suárez FC, Gonzalez-Fajardo JA, Monreal BM. Registro informatizado dela enfermedad tromboembólica en España (RIETE). Objetivo, métodos y resultados preliminares. Rev Clin Esp. 2003; 203:68-73.

10. Santos-Sancho J.M., Gil-Prieto R., Álvaro-Meca A., Gil-de Miguel A. Epidemiología de las hospitalizaciones causadas por rubéola en la población general en España (1997-2006). Anales SisSan Navarra [revista en la Internet]. 2010 Abr [citado 2012 Sep 27] ; 33(1): 65-70. Disponible http://scielo.isciii.es/scielo.php?script=sci_arttext\&pid=S1137-66272010000100007\&lng=es. http://dx.doi.org/10.4321/S1137-66272010000100007

11. López Izquierdo Raúl, Asensio Villahoz Paula, Vicente Vírseda Juan Antonio, González Manzano Isabel, Udaondo Cascante $\mathrm{M}^{\mathrm{a}}$ Antonia. Patología tuberculosa 
atendida en urgencias a través del análisis del CMBD de hospitalización en el área de Valladolid Oeste (2002-2006). Rev. Esp. Salud

Publica [serial on the Internet]. 2009 Apr [cited 2012 Sep 27] ; 83(2): 279-290. Available from: http://www.scielosp.org/scielo.php?script=sci_arttext\&pid=S113557272009000200011\&lng=en. http://dx.doi.org/10.1590/S1135-57272009000200011

12. Peña-Rey I, Martines de Aragón MV, Villaverde Hueso A, et al. EPIDEMIOLOGÍA DE LA VARICELA EN ESPAÑA EN LOS PERÍODOS PRE Y POST VACUNACIÓN. Rev Esp Salud Pública. 2009; 83:711-724 N. 5 - Septiembre-Octubre.

13. Corral Baena S, Guerrero Aznar MD, Beltrán García M, et al. Utilización del CMBD como herramienta para la detección de acontecimientos adversos a medicamentos. FARM HOSP (Madrid). 2004; 28(4):258-265.

14. Barba R, Losa JE, Guijarro C, et al. Fiabilidad del conjunto mínimo básico de datos (CMBD) en el diagnóstico de la enfermedad tromboembólica. Med Clin (Barc). 2006; 127(7):255-7.

15. Institute of Medicine. Reliability of hospital discharge records. Washington DC. National Academy of Sciences. 1977.

16. Institute of Medicine. Realiability of hospital discharge survey data. Washington DC: National Academy of Sciences. 1980.

17. Institute of Medicine. Realiability of hospital discharge abstracts. Washingto DC: National Academy of Sciences. 1997.

18. Green J, Wintfeld N. How accurate are hospital discharge data for evaluating effectiveness of care. Med Care. 1993; 31:719-31.

19. Hsia DC, Ahern C, Ritchie BP, et al. Medicare reimbursement accuracy under the prospective payment system, 1985 to 1988. JAMA. 1992; 268:896-9.

20. Yetano J, Izarzugaza I, Aldasor E, et al. Calidad de las variables administrativas del Conjunto Mínimo Básico de Datos de Osakidetza-Servicio Vasco de Salud. Rev Calidad Asistencial. 2008; 23(5):216-21.

21. Assaf AR, Lapane K, McKenney J, et al. Possible influence of the prospective payment system on the assignment of discharge diagnoses for coronary heart disease.N Engl J Med, 1993; 329:931-5.

22. Malenka DJ, McLerran D, Roos $\mathrm{N}$, et al. Using administrative data to describe casemix: a comparison with the medical record. J Clin Epidemiol. 1994; 47:1027-32.

23. Peiro S, Librero J. Evaluación de la calidad a partir del conjunto mínimo de datos básicos al alta hospitalaria. Rev Neurol. 1999; 29:651-61.

24. Reyes A, González A, Rojas MF, et al . Grupo de Estudios de la Calidad Asistencial (GRECA). Los informes de alta hospitalaria médica pueden ser una fuente insuficiente de información para evaluar la calidad de la asistencia. Rev Clin Esp. $2001 ; 201(12): 685-9$.

ISSN 1695-6141

๑ COPYRIGHT Servicio de Publicaciones - Universidad de Murcia 